\title{
QUOTATIONS FROM AGB98
}

- Actually, it is a C-star model which we used for O-rich stars.

(B. Plez, answering to a question about the selection of models that so nicely agree with the observations.)

- We are still far from deriving stellar parameters from such modelling, but we are certainly getting closer to it.

(B. Plez, this volume, p. 81)

- We have to burn the haystack to get the needle.

(K. Lodders, on what happens with stardust in the lab.)

- Reaction rates are measured at room temperature, and then extrapolated at $30 \mathrm{~K}$ ! (A. Glassgold)

- Each observation amounted to one hour of ISO time; some yielded interesting results, others, not.

(H. Izumiura)

- The imagination of all of us is very large, and the constraints from observations are very scarce.

(B. Lopez)

- All $R$ stars appear to be single. Thus, since it is not normal that all objects in a population are single, they must all have been binaries in the past.

(C. Tout)

- I didn't get the observing time and thus had to be a bit more creative, and so... I looked at the data again!

(Y.K. Ng)

- I made a mistake, you have twenty more minutes.

(A. Omont, session chairman, to reviewer A. Zijlstra)

- Non-sphericity doesnot mean chaos.

(M. Jura) 


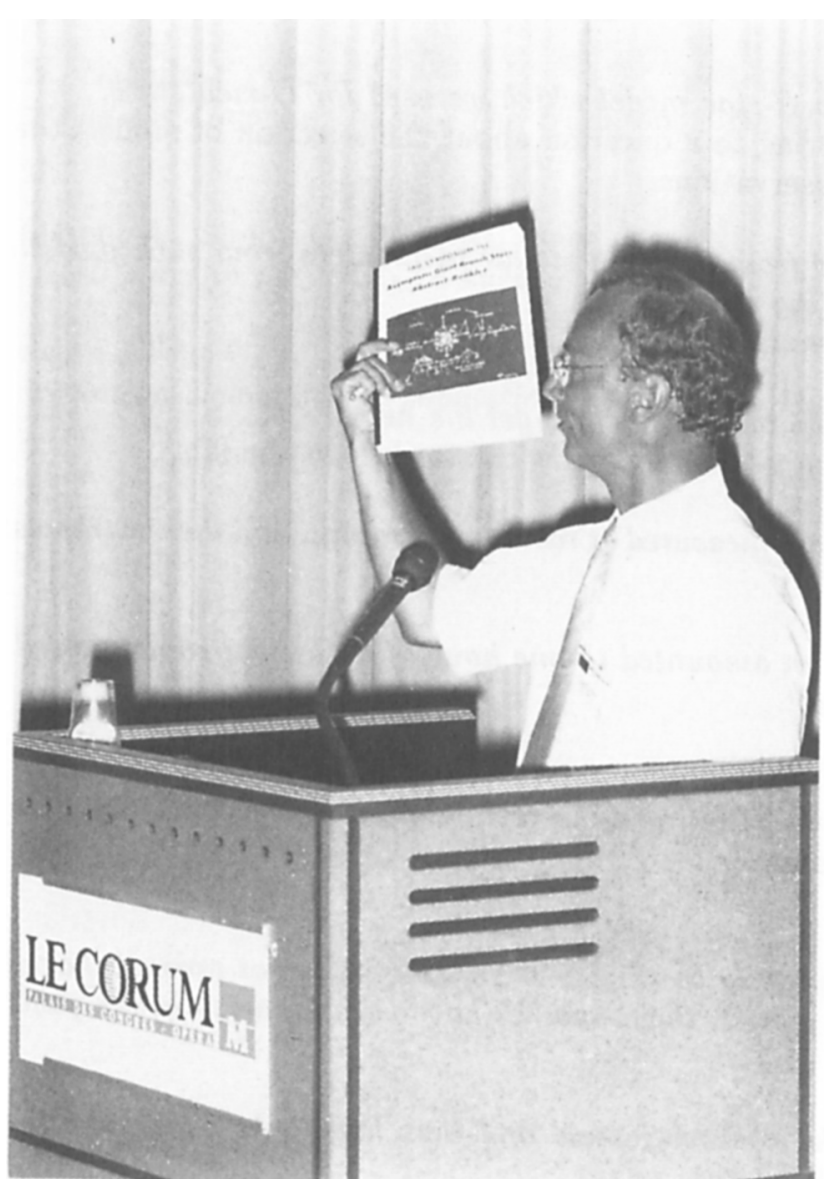

Christoffel Waelkens publicizing the ABSTRACT Booklet 


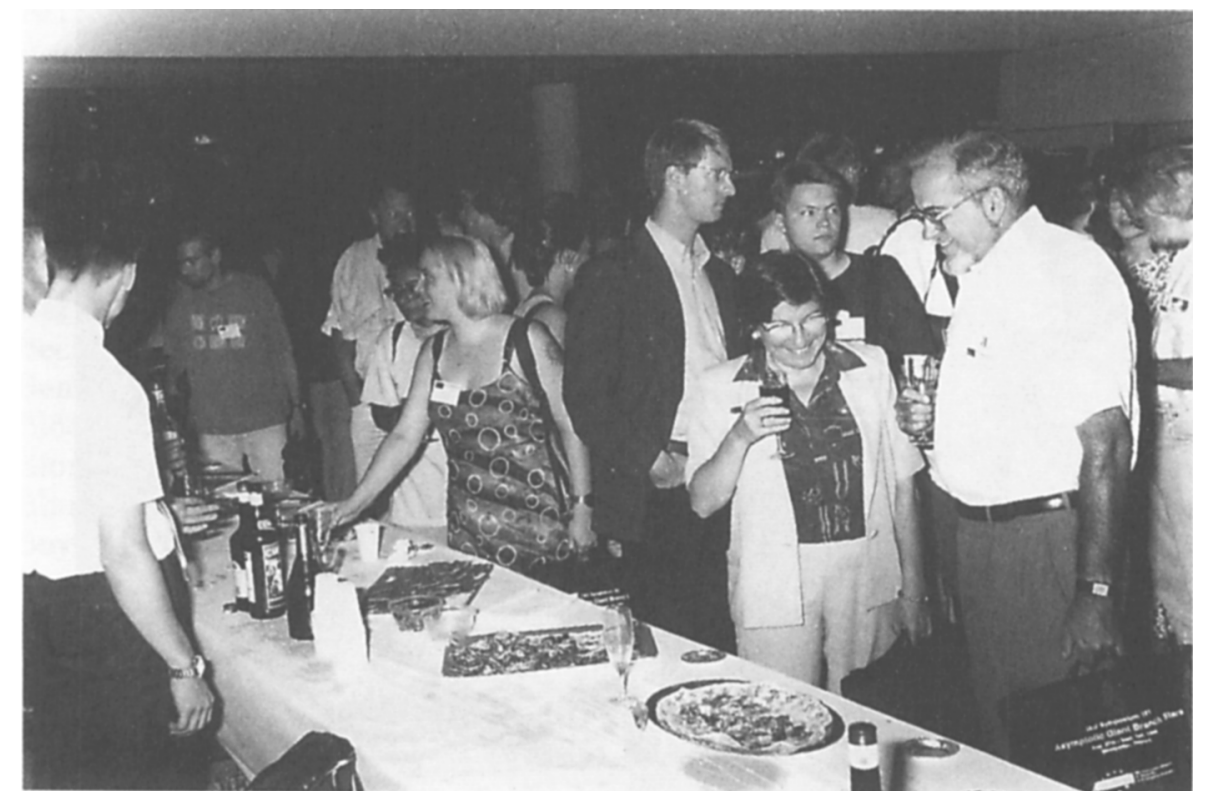

Janet Mattei and Bob Wing enjoying the buffet 


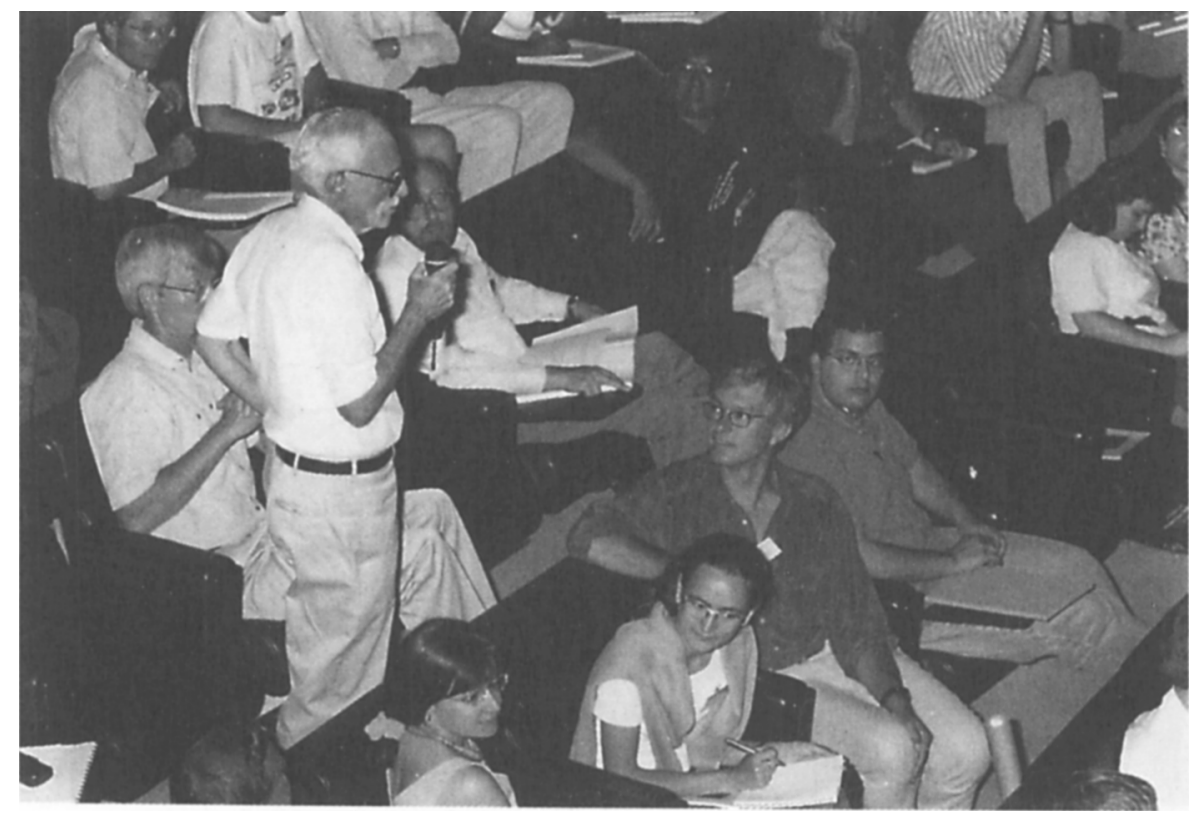

Alfred Glassgold, Ariane Lançon, Ulli Käufl, Mustapha Mouhcine, etc. 